\title{
“LA AUSENCIA DE AUTONOMÍA JURISDICCIONAL AERONÁUTICA EN LA ARGENTINA REPRESENTA UN SERIO RIESGO PARA LOS DERECHOS DEL TURISTA?”: Comentario a la Sentencia de la Cámara Federal de Apelaciones de Córdoba, Secretaría Civil II, Sala A, en los autos “L.O.H. c/ LATAM s/DAÑOS Y PERJUICIOS” de fecha 26 de septiembre del año 2018
}

\begin{abstract}
“THE ABSENCE OF AERONAUTICAL JURISDICTIONAL AUTONOMY IN ARGENTINA AS A SERIOUS RISK FOR THE RIGHTS OF TOURIST?”: Comentario a la Sentencia de la Cámara Federal de Apelaciones de Córdoba, Secretaría Civil II, Sala A, en los autos “L.O.H. c/ LATAM s/DAÑOS Y PERJUICIOS” de fecha 26 de septiembre del año 2018
\end{abstract}

\author{
Ab. ERNESTO FRONTERA - Ab. GUADALUPE HIDALGO - \\ Ab. Prof. HUGO RIVAROLA \\ Derecho de la Navegación, Transporte y Comunicaciones Cátedra “C” \\ Facultad de Derecho de la Universidad Nacional de Córdoba (Argentina)
}

\section{Resumen}

Inexistencia de un fuero especializado en materia de Navegación Aérea. Derechos de los turistas. Competencia e incompetencia federal. Seguridad Jurídica y garantías constitucionales.

Palabras Claves: Derecho del Turismo. Pasajero aeronáutico. Competencia Federal. Responsabilidad del Transportador Aéreo. Operaciones de embarco y desembarco de aeronaves.

\section{Abstract}

Inexistence of a jurisdiction specialized in aerial navigation. Rights of tourists. Federal competence and incompetence. Legal Security and constitutional guarantees.

Key Words: Travel Law. Aeronautical passenger. Federal Competence. Responsibility of the Air Transporter. Aircraft embarkation and disembarkation operations.

Fecha de recepción: 15 de marzo de 2019

Fecha de aprobación: 22 de mayo de 2019 


\section{INTRODUCCIÓN}

Dado el estadío actual del Derecho de la Navegación Aérea, nos preguntamos, si la falta de un fuero especializado en la materia no resulta una grave carencia en el ordenamiento jurídico argentino, sobre todo, ante la proliferación de fallos y argumentaciones contradictorios en temas de suma importancia, tales como el que nos convoca en el particular; la competencia o incompetencia federal, y si esta situación genera o puede generar la afectación de los derechos del turista.

En ese contexto, debemos tener presente que se encuentran en juego principios liminares del Derecho en materia procesal, entre otros, el principio "iura novit curia" y el derecho de peticionar ante las autoridades.

En lo particular y concreto, se analizará un caso donde se declara la incompetencia federal, con un voto en disidencia.

En esa dirección, creemos necesario realizar una exposición previa de los lineamientos básicos de la competencia federal en la República Argentina.

\section{DESARROLLO}

\section{a. El origen constitucional de la Competencia Federal y su carácter de limitada y excepcional}

En este punto resulta pertinente establecer algunos lineamientos básicos en relación a la competencia federal. En esta materia, seguiremos en lo trascendental, la obra de Claudio Daniel Gómez titulada "Competencia Federal" ". En primer lugar, destacamos el origen constitucional, lo cual significa, que al organizarla el Congreso no ha ejercido una facultad sino que ha cumplido una obligación, de modo que no podría suprimirla por una ley ni alterar su competencia sin violar el principio constitucional.

Esto se relaciona íntimamente con el sistema político federal de la República Argentina, por el cual las provincias son entes políticos preexistentes que constituyeron el gobierno central y al cual le delegaron materias.

Así, el origen constitucional de la jurisdicción federal se debe a que su actuación depende en todos los casos del articulado de la Carta Magna que el Congreso Nacional puede reglamentar. Según la Corte Suprema de Justicia de la Nación, el congreso puede consagrar excepciones a la competencia federal, cuando no exista un interés federal de entidad a proteger pero no ampliarla. Un ejemplo de restricción legislativa puede verse en materia de juicios universales y concursos; prórroga de la competencia federal ratione personae a los tribunales provinciales, entre otros) (fallos: 99-408;152-346, entre otros).

\footnotetext{
${ }^{1}$ GOMEZ, CLAUDIO DANIEL.Competencia Federal . Buenos Aires : Mediterranea.
} 
De lo expuesto resulta que la competencia federal es limitada a lo prescripto por los arts. $116^{2}$ y $117^{3} \mathrm{CN}$, y que se trata de una competencia de excepción, esto es, requiere mención expresa, es de interpretación restrictiva y no se pueden aplicar las reglas de la analogía.

\section{b. El Interés Federal Afectado}

De manera genérica y abstracta podemos decir que el justiciable debe invocar y probar un interés federal para justificar la apertura del fuero federal, pero, ¿qué rasgos debe tener este interés? La doctrina reza que debe ser cierto, real, objetivo, legítimo, concreto, y con suficiente entidad.

Es muy usual encontrar fallos donde se declara, expresa o implícitamente, la falta de entidad o envergadura en el interés federal invocado y el caso que comentamos no es la excepción. Véase infra en el voto de la mayoría.

Por otro lado, la Competencia Federal puede dividirse en razón de la materia (ratione materiae), en razón de las personas (ratione personae), en razón del lugar (ratione loci), en razón del grado (ratione gradius) y en razón del valor (ratione pretium). El fallo que se comenta presenta relación con la competencia "ratione materiae", por lo cual, nuestro análisis se circunscribe a ella.

\section{c. La competencia federal en relación a la materia (ratione materiae)}

En este caso el conflicto versa en forma directa y principal sobre el derecho federal. Esto quiere decir, que la causa predominante debe ser la federal. Esto, a su vez, se relaciona íntimamente con el rasgo de entidad que debe tener el interés federal.

Siguiendo a GOMEZ, en la obra citada, las "fuentes" del derecho federal son: 1) La Constitución Nacional, 2) leyes federales y sus decretos reglamentarios, 3) Tratados con las potencias extranjeras, 4) Causas de almirantazgo y jurisdicción marítima y, 5) Actos administrativos del gobierno nacional.

Se trata de una competencia apelada, es decir, se atribuye a los jueces federales de grado y es improrrogable a los tribunales provinciales. Nótese, como la improrrogabilidad de la competencia federal en razón de la materia puede agravar la situación del justiciable ante un panorama de por sí sombrío respecto a la entidad que debe tener el interés federal afectado.

2 Artículo 116 Constitución Nacional: Corresponde a la Corte Suprema y a los tribunales inferiores de la Nación, el conocimiento y decisión de todas las causas que versen sobre puntos regidos por la Constitución, y por las leyes de la Nación, con la reserva hecha en el inciso 12 del artículo 75; y por los tratados con las naciones extranjeras; de las causas concernientes a embajadores, ministros públicos y cónsules extranjeros; de las causas de almirantazgo y jurisdicción marítima; de los asuntos en que la Nación sea parte; de las causas que se susciten entre dos o más provincias; entre una provincia y los vecinos de otra; entre los vecinos de diferentes provincias; y entre una provincia o sus vecinos, contra un Estado o ciudadano extranjero.

${ }^{3}$ Artículo 117 Constitución Nacional: En estos casos la Corte Suprema ejercerá su jurisdicción por apelación según las reglas y excepciones que prescriba el Congreso; pero en todos los asuntos concernientes a embajadores, ministros y cónsules extranjeros, y en los que alguna provincia fuese parte, la ejercerá originaria y exclusivamente. 


\section{d. Hechos}

El Aeropuerto Internacional Ingeniero Ambrosio Taravella de la ciudad de Córdoba, Argentina, se encuentra concesionado a la empresa Aeropuertos Argentina 2000 S.A, en virtud del Decreto Nro. 163/98 y forma parte del Sistema Nacional de Aeropuertos.

El caso jurisprudencial en cuestión consiste en un pasajero turista de la empresa LATAM, Número de Vuelo 952, proveniente de Estados Unidos de América, el cual contaba con una escala en la ciudad de Santiago de Chile, y tenía por destino final la ciudad de Córdoba. Luego del aterrizaje de la aeronave en el Aeropuerto de la Ciudad de Córdoba, encontrándose bajo expresas directivas y custodia de la empresa transportadora, descendió de la misma, a efectos de concurrir a las dependencias de migraciones y aduana argentinas para formalizar su ingreso al país. En ese momento, el Aeropuerto Ingeniero Ambrosio Taravella se encontraba en reparación edilicia, y por tanto, el pasajero tuvo que ascender por una rampa bastante "precaria”, (ya que se encontraba en desnivel y en plano inclinado con relación al camino en el que venía circulando), y fue allí en donde perdió el equilibrio, cayó al suelo, e impactó contra el mismo con la región sacro coxígea. El pasajero expresó que no fue asistido por ningún servicio de emergencia, sino que por el contrario, la transportadora sólo le suministró una silla de ruedas y un maletero. En base a una serie de estudios médicos realizados en la ciudad de Córdoba, se le diagnosticó "traumatismo de cadera derecha con quebradura de pelvis”, y con posterioridad se le determinó una incapacidad residual post traumática parcial y permanente del $40 \%$ de la total obrera.

Por los hechos expuestos, el pasajero interpuso una demanda de daños y perjuicios contra la empresa de Transporte Aéreo LATAM, por la suma total de \$255.542,53, más intereses y costas, en los Tribunales Federales de la Provincia de Córdoba. En un primer momento, el Fiscal interviniente dictaminó que no resultaba procedente la demanda, atento a no resultar, el fuero Federal, competente en razón de la materia para la resolución de dicho pleito. El Juez Federal Nro. 1 adhirió a dicho dictamen, se declaró incompetente sin mediar sustanciación en su decreto, y archivó las actuaciones. En virtud de dicho proveído, la parte actora interpuso recurso de reposición con apelación en subsidio, fundando su agravio en que el Juez interviniente, “adhirió al dictamen del Fiscal sin sustanciación, violentando los principios de contradicción, congruencia y defensa".

El apelante, manifestó también que lo resuelto, “carecía de argumentación y motivación, y que al mismo tiempo había un desconocimiento de los argumentos expuestos en la demanda sobre la competencia territorial y en razón de la materia al versar la misma sobre los daños y perjuicios de un pasajero y en relación a las normas que involucran la aplicación de la legislación Aeronáutica, tanto el Convenio de Montreal de 1999 y el Código Aeronáutico Argentino". Afirmó también, que en el caso concreto existe una relación contractual a través del billete de pasaje aéreo, de lo cual se desprende una responsabilidad del transportador, y que por lo tanto, su entendimiento corresponde a la Justicia Federal.

\section{e. Fundamentos del Apelante}

En el recurso de reposición y apelación en subsidio, el agraviado se manifiesta en contra del dictamen fiscal en relación al punto donde se desestima la apertura del fuero federal por la falta de encuadramiento de la causa en el art. 116 de la Constitución Nacional. 
Ahora bien, en nuestra opinión, el apelante vierte una serie de argumentos, de los cuales, algunos nos parecen débiles y otros pertinentes para la resolución del caso.

En principio, toda la argumentación doctrinal y jurisprudencial referida al art. 198 del Código Aeronáutico Argentino ${ }^{4}$ nos parece complementaria, por la sencilla razón, que el factum nos revela un vuelo internacional y, por lo tanto, el contrato de transporte aéreo internacional de pasajeros queda regulado en el ámbito del Convenio de Montreal de 1999, en vigencia en la República Argentina a partir de Enero de 2010.

En cambio, consideramos de principal y de mayor entidad y pertinencia, la referencia a la ley federal $n^{\circ}$ 13.998/50, regulatoria de la Organización de la Justicia Nacional. En efecto: el art. 55 inc. b, en forma categórica, determina la competencia federal para los "hechos, actos y contratos concernientes a los medios de transporte terrestre...; y a los regidos por el derecho de la navegación y por el derecho aeronáutico”.

Si bien esta ley constituye una fuente formal de derecho federal, para que lo dispuesto en la disposición transcripta pueda subsumirse en el art. 116 C.N, es necesario tener en cuenta una de las atribuciones que la Carta Magna concede al Congreso Nacional, esto es, la denominada “cláusula comercial” (art. 75 inc. 13 C.N.); la cual implica la atribución de regular el comercio inter-jurisdiccional, ya sea internacional o interprovincial.

Nótese, que a diferencia de la actividad marítima, la actividad aeronáutica no está contemplada de manera expresa por el art. 116 C.N, sin embargo, en ambos casos, es necesario que estemos frente a una actividad incluida en una atribución delegada por las provincias a la Nación. De lo contrario, el interés federal sería, como mínimo, débil.

Es justamente por lo expuesto, que el apelante, siguiendo a Palacio, llega a similar argumentación cuando expresa que: “Asevera Palacio que: “El art. 55 inc. b de la ley No 13.998 atribuye a los jueces federales con asiento en las provincias competencia para conocer de los hechos, actos y contratos regidos por el derecho aeronáutico" agregando que es requisito de esa competencia, "que se trate de aeronavegación internacional o interprovincial, es decir, que se realiza respectivamente, entre un aeródromo de la república y otro extranjero..."5

Por último, tenemos que destacar un problema de interpretación que nos parece decisivo para la resolución del particular; los artículos de la Constitución Nacional donde se determina la competencia federal, tanto originaria y exclusiva de la CSJN, como la apelada (arts. 116 y 117 C.N.), han sido reglamentados en el año 1863 por la Ley 48 de Jurisdicción y Competencia de los Tribunales Nacionales.

En su art. 2, la Ley 48 reglamenta la competencia apelada, en virtud de la cual, "los jueces nacionales de sección conocerán en primera instancia de las causas siguientes:”

${ }^{4}$ Articulo 198 Código Aeronáutico Argentino: Corresponde a la Corte Suprema de Justicia y a los tribunales inferiores de la Nación el conocimiento y decisión de las causas que versen sobre navegación aérea o comercio aéreo en general y de los delitos que puedan afectarlos.

${ }^{5}$ PALACIO, LINO ENRIQUE. Manual de Derecho Procesal Civil. Buenos Aires : Lexis- Nexis - Abeledo Perrot. 
De manera general, el inc. 1 reza "las que sean especialmente regidas por la Constitución Nacional, las leyes que hayan sancionado y sancionare el Congreso y los tratados públicos con naciones extranjeras".

Es clásica la clasificación doctrinal de las leyes en: 1) locales, 2) comunes y 3) federales. En ese sentido no cabe duda, que este inciso descarta la apertura del fuero federal en causas de derecho común (art. 75 inc. 12 C.N), y de derecho local, por no ser especialmente regidas por la Constitución Nacional. En cambio, se refiere claramente, a leyes federales en sentido amplio (Constitución, ley, etc.).

En los incisos 7, 8 y 9, el Congreso Nacional, de manera casuística, reglamenta causas relacionadas con la actividad marítima, que por su mayor o menor gravedad, generan o podrían generar el entorpecimiento de la actividad.

A nuestro modo de ver, estos incisos son meramente ejemplificativos, y los que, por un prurito formal, quieran aferrarse al carácter excepcional de la competencia federal, se verán en el escollo insalvable de cómo trasladar dichos ejemplos al ámbito aeronáutico. Por lo demás, semejante interpretación desnaturalizaría el inciso primero, que es el verdadero faro para el intérprete.

En el año 1950, el Congreso Nacional dicta la ley 13.998 de Organización de la Justicia Federal y como ya se expresó, sin entrar en casuística alguna, establece la competencia federal en hechos, actos y contratos regidos por el derecho marítimo y aeronáutico (art. 42 inc b, y art. 52 inc. b). De una sana hermenéutica debemos colegir que esta ley tiene preeminencia frente a la ley 48, ya que es posterior.

Por eso, coincidimos con el agraviado, en que resulta abstracta la desestimación de la competencia federal con base en el art. 2, ley 48.

\section{f. El Voto de la Mayoría}

El Sr. Juez Eduardo Ávalos y la Sra. Juez Graciela S. Montesi, de manera coincidente con el dictamen del Fiscal General, votaron desestimando la pretensión de apertura del fuero federal por falta de encuadramiento de la causa en el art. 116 de la Constitución Nacional.

Si bien se afirma que, según las circunstancias fácticas, "no se ha visto conculcados intereses federales alguno", en rigor, existen dos lineamientos argumentales diferentes, el primero implícito, y el segundo expreso.

Siguiendo el orden expositivo del preopinante, en primer lugar sostiene, que el interés federal invocado no tiene la suficiente entidad y, en segundo lugar, que ni siquiera hay interés federal, ya que el hecho dañoso aconteció fuera de la zona de desembarque, ergo, se trata de un caso de derecho común.

En rigor, el orden sería el siguiente: no existe interés federal por tratarse de una causa de derecho común, pero si fuera federal, el interés federal invocado no tiene envergadura, ergo, se lo considera inexistente.

No resulta lo mismo en el plano argumental, que a los ojos del intérprete, el interés federal sea débil, que lisa y llanamente no exista. Si fuera lo mismo, nos preguntamos el para qué 
de la doctrina que, de manera sistemática, afirma que el interés federal invocado debe ser de entidad.

Lo expresado se corrobora cuando se dice "no advierto que el hecho denunciado, guarde vinculación con la seguridad, el comercio, los intereses de la aeronavegación o con las normas federales de derecho aeronáutico".

En relación a este primer argumento, sostenemos que, si cada reclamante, a falta de reglas legislativas o judiciales (fallos plenarios) tendría que "adivinar" cuando un interés federal presenta la suficiente entidad como para justificar la apertura del fuero federal, la seguridad jurídica y el derecho de peticionar ante las autoridades podrían verse afectados, máxime cuando la competencia federal en razón de la materia es improrrogable a los tribunales provinciales.

Una situación admisible sería que el Congreso Nacional restrinja la competencia federal en orden a la falta de envergadura del interés federal invocado, siempre con la anuencia expresa o tácita del último guardián e intérprete de la Constitución Nacional, la CSJN., pero otra muy distinta, que ello dependa del mero arbitrio judicial.

Para nosotros, si el hecho dañoso aconteció durante la vigencia del contrato aeronáutico y se trata de una actividad inter-jurisdiccional, el interés federal debe considerarse siempre de entidad, o lo que es lo mismo, es el predominante.

Desde ya aclaramos, que desde el punto de vista de la Filosofía del Derecho, esta interpretación no es perfecta. La realidad presentará casos difíciles o dudosos donde habrá tantos argumentos a favor como en contra a la hora de determinar la suficiente entidad del interés federal. Pero afirmar lo contrario, además de brindar una tremenda discrecionalidad judicial, es poner en serio riesgo principios rectores que hacen a la Seguridad Jurídica, y por lo tanto, se podrían afectar gravemente derechos subjetivos. Por lo tanto, en el contexto actual, entendemos que esta interpretación es la que mejor armoniza con el objeto formal del Derecho: la Justicia.

Por lo demás, ni la Constitución Nacional, ni la ley 13.998/50 exigen expresamente el requisito de entidad suficiente.

Respecto al segundo argumento, si se trata de un caso de derecho común, la regla es que corresponde la competencia provincial por imperio del art.75 inc. 12 C.N.

$\mathrm{Si}$, como piensan los vocales del voto mayoritario, el daño se produjo fuera de la zona de desembarque, seríamos contestes en coincidir en que, por ser un caso de derecho común, no corresponde la justicia federal.

Pero justamente, para nosotros y según lo probado en el expediente, el daño se produjo en la zona de desembarque, y considerando que se trataba de un vuelo internacional proveniente de los Estados Unidos de América, y que tenía por destino final la Ciudad de Córdoba de donde es oriundo el pasajero, corresponde tener en cuenta a la hora de fallar, la normativa internacional que rige la materia. Por tal motivo, el Juez Federal debió aplicar el Convenio de Montreal de 1999, ratificado por nuestro país, y el cual, en su Capítulo Tercero, titulado Responsabilidad del Transportista y Medida de la Indemnización por Daño, Artículo Nro. 17 Muerte y lesión de los pasajeros. Daño al equipaje, reza: “1. El transportador es responsable del daño causado en caso de muerte o lesión corporal de un pasajero por la sola razón de que el accidente que causó el 
daño se haya producido a bordo de la aeronave o durante cualquiera de las operaciones de embarque o desembarque."

El maestro Videla Escalada nos enseñó que la responsabilidad del operador aeronáutico “...comprende a todos los supuestos en que un sujeto de derecho debe responder frente a la comunidad o frente a otra persona en virtud de hechos o situaciones en los se ha producido algún daño y en los que el ordenamiento jurídico considera que ha habido una manifestación antijurídica...” 6

También, podemos citar a Goedhuis ${ }^{7}$, que fue quien luego de realizar todo un análisis interpretativo, teniendo en cuenta la Convención de Varsovia de 1929 y lo tratado en el Segundo Comité de CITEJA, llegó a una conclusión respecto de qué se entiende por "operaciones de embarque y desembarque".

El autor, refiere que son aquellas maniobras realizadas desde que el pasajero sale del edificio del aeropuerto a tomar el avión, hasta que entra al edificio del aeropuerto de destino. Considera, que si bien esta definición tiene sus fallas, resulta la más acertada y debería distinguirse dos situaciones. Por un lado, la primera situación sería, si el pasajero es acompañado a tomar el avión (o abandonarlo) con un dependiente del transportador, y es allí cuando ocurren los daños. En este caso, el transportador no será responsable si prueba haber tomado las medidas necesarias para que el daño no ocurriera. La otra situación sería si el pasajero no es acompañado por un dependiente del transportador, ni tampoco tiene posibilidades de hacerlo, ni tampoco de colocar el avión cerca. Para dicho autor, en este caso significaba que el transportador había tomado las medidas necesarias, pese a su imposibilidad, y por lo que no sería responsable. Por tanto, Goedhuis afirma, que la responsabilidad de transportador empieza cuando el pasajero pisa la loza de fin para dirigirse al avión del transportador a los fines de realizar el viaje y termina cuando el pasajero abandonó la loza del de destino.

Sin perjuicio de ello, recurriendo al derecho comparado, resulta importante citar jurisprudencia internacional, precisamente las consideraciones de los tribunales norteamericanos, los cuales continúan fieles a la jurisprudencia iniciada por el caso "Buronocore v. Trans World Airlines, Inc." " que fue en donde se sentaron las bases para establecer una serie de factores que deben tomarse en cuenta para determinar si los hechos ocurrieron "en el curso de las operaciones de embarque o desembarque". Estos factores son: a) la actividad del pasajero en el momento del accidente, b) las restricciones impuestas por la empresa aérea a su movimiento, c) la inminencia del momento de embarque o desembarque, $y$, d) la proximidad física del pasajero a la puerta de salida/entrada. ${ }^{9}$

\footnotetext{
${ }^{6}$ VIDELA ESCALADA, FEDERICO.Manual de Derecho Aeronautico. Buenos Aires : Zavalia, 2007.

${ }^{7}$ MARTINEZ MARTINEZ, ESUTAQUIO. Jurisprudencia Internacional sobre la Convencion de Valsovia de 1929, referente a la responsabilidad civil del porteador aéreo. [aut. libro] Goedhurs. Santiago de Chile : Editorial Juridica de Chile, 1962.

${ }^{8}$ Buronocore v. Trans World Airlines, Inc. 900 F.2d 8, 10, 2d Cir.. Estados Unidos : United States Court of Appeals, Second Circuit, 1990.

${ }^{9}$ CAPALDO, GRISELDA. cedaeonline.com. [En línea] Powered by WordPress.com, 11 de 07 de 2013. [Citado el: 05 de 03 de 2019.] https://cedaeonline.com.ar/2013/07/11/nueve-anos-de-vigencia-del-convenio-demontreal-de-1999-su-interpretacion-jurisprudencial-respecto.
} 
Por todo lo expuesto, en virtud de las pruebas agregadas en autos, nos parece incorrecta la apreciación de los hechos realizada por el Tribunal interviniente, y como consecuencia de ello, el derecho aplicable y la resolución arribada. Por tal motivo, y como se dijo anteriormente, el pasajero se encontraba realizando "operaciones de desembarco" de la aeronave, y bajo las órdenes y custodia del transportador, quien debió tomar las medidas necesarias para evitar que se produjeran los daños acaecidos.

\section{g. El Voto en Disidencia}

A diferencia de la mayoría, el Sr. Juez Ignacio María Vélez Funes entendió que: el hecho dañoso ocurrió en la zona de desembarque, y por lo tanto, durante la vigencia del contrato aéreo internacional de pasajeros.

Debido a la falta de argumentación al respecto, pareciera, que ese solo hecho determina, sin más, la competencia federal.

Coincidimos en lo atinente a que no se trata de un caso de derecho común, por haber ocurrido el hecho en la zona de desembarque y por las explicaciones vertidas supra.

Ahora bien, como previamente manifestamos al comentar los fundamentos del apelante, no se debe olvidar que la competencia federal es limitada a los art. 116 y 117 C.N., y en razón de eso, que el interés federal debe ser el predominante.

A nuestro juicio, esto acontece cuando el transporte es interjurisdiccional (art.75 inc. 13 C.N.), como sucede en el caso en análisis, pero creemos que este argumento es vital a la hora de resolver el caso, no bastando que el hecho suceda durante la ejecución de un contrato de transporte.

Debido a esta interpretación, es que si se trata de un vuelo intra-provincial, la discusión respecto de la competencia federal es una cuestión altamente polémica.

\section{CONCLUSIONES}

A modo de conclusión, podemos hacer las siguientes afirmaciones y sugerencias: en primer lugar, nos parece errónea la interpretación de la mayoría en orden a una falta de encuadramiento del caso en el art. 116. C.N. Existe una ley federal, la número 13998/50, que de manera categórica, determina la competencia federal en materia aeronáutica, la cual ha sido ignorada por completo al resolver el caso en cuestión. Si bien lo que se pretende es un resarcimiento por daños y perjuicios, estamos en presencia de una causa predominantemente federal, por tratarse de un hecho acaecido durante la vigencia de un contrato de transporte aéreo de pasajeros interjurisdiccional; materia atribuida al Congreso de la Nación (art. 75 inc 13 C.N.).

También hemos podido visualizar, que se trata de uno de los tantos casos donde, en forma palmaria, un turista puede verse afectado en sus derechos subjetivos, ya que no sólo se violenta el principio de contradicción, congruencia y de defensa de raigambre constitucional, sino que también privó al pasajero de obtener una respuesta a los daños físicos sufridos. Como dato de 
color, entendemos que el actor podría haber escogido otra opción; demandar al explotador de servicios aeroportuarios por la falta de prestación de servicio de emergencia al momento que ocurrieron los hechos, como así también, por las precarias condiciones de seguridad operacional para el desembarco de pasajeros (sobre todo de un vuelo internacional), en la plataforma del Aeropuerto. No obstante, al tratarse de una responsabilidad de derecho común, correspondería el Fuero ordinario, situación que corrobora la incertidumbre reinante para quienes pretenden ejercer sus legítimos derechos de resarcimiento.

Por último, creemos que la falta de un fuero especializado representa un serio riesgo para los derechos del turista; la confusión, la despreocupación y falta de conocimientos técnicos que se evidencia en el fuero civil y comercial, a la hora de abordar temas aeronáuticos, es un dato de la realidad. Sin pretender una relación causal, y como una alternativa de cambio, entendemos que la creación de un fuero especializado, al menos, generaría una visión de unidad y una necesidad de repuesta de mayor calidad, coherencia y uniformidad.

\section{SUMARIO DE LA SENTENCIA}

"Los presentes autos llegan a conocimiento y decisión de este Tribunal en virtud del recurso de apelación en subsidio de la reposición interpuesta por la parte actora, en contra del proveído de fecha 7 de noviembre de 2017, dictado por el señor Juez Federal $\mathrm{N}^{\circ} 1$ de Córdoba, que adhiriendo a lo dictaminado por el fiscal interviniente, declaró la incompetencia de la justicia federal para intervenir en la presente causa, disponiendo el archivo de la misma.”

"En su libelo recursivo, el apelante se agravia en tanto el Juez de la causa adhirió sin sustanciación al dictamen fiscal, violentando los principios de contradicción, congruencia y defensa. En ese marco, señala que lo decidido carece de argumentación y motivación al mismo tiempo que desconoce los argumentos expuestos en la demanda sobre la competencia territorial y en razón de la materia al versar la misma sobre los daños y perjuicios sobre un pasajero y en relación a las normas que involucran la aplicación de la legislación Aeronáutica, tanto el Convenio de Montreal de 1999 y el Código Aeronáutico Argentino. Afirma que en el caso concreto existe una relación contractual a través del billete de pasaje aéreo, de lo cual se desprende una responsabilidad del transportador, cuyo entendimiento corresponde a la Justicia Federal. Cita doctrina y jurisprudencia en apoyo de su postura (fs. 99/100 vta.).”

"Entonces, sin desconocer el infortunio bajo análisis ni sus consecuencias, advierto que el mismo no engarza en la inteligencia que corresponde asignarle a la normativa aeronáutica invocada para sostener la competencia de excepción en la medida de que nos encontramos ante una pretensión resarcitoria de derecho común, que a la luz de las consideraciones dadas -como ya señalé- en modo alguno afectó los intereses sobre los que aquellas disposiciones legales mencionadas se proyectan jurídicamente.”

"Tampoco se da la competencia en razón de las personas, toda vez que tanto el actor como la demandada son vecinos de esta ciudad de Córdoba (arts. 2 inc. 2 de la Ley 48). “

"Por todo lo expuesto, conforme lo dictaminado por el Fiscal General, entiendo que corresponde rechazar el recurso de apelación incoado y, en consecuencia, confirmar el proveído 
de fecha 7 de noviembre de 2017 dictado por el señor Juez Federal $N^{\circ} 1$ de Córdoba en cuanto declaró en definitiva la incompetencia del fuero federal por remisión a los fundamentos vertidos en el dictamen del representante del Ministerio Público Fiscal de la instancia. Sin costas atento la falta de contradictorio.”.

"La primera pauta comprende los acontecimientos dañosos producidos a bordo de la aeronave. Esta expresión resulta relativamente sencilla, más dificultoso es el problema de la determinación del otro supuesto mencionado en el Convenio, ya que el periodo de transporte aéreo comprende el embarque, el vuelo y el desembarque...” Así continua su análisis diciendo que “...El criterio más equitativo para resolver el problema debe tener en mira el tiempo en que el pasajero está a disposición del transportista y bajo su control, con el fin de ser trasladado al punto de destino, lapso que se cubre con la mención de las operaciones de embarque y desembarque. El embarque comienza cuando el pasajero penetra en el terreno de partida, ya que el pasajero hasta ese momento o se somete efectivamente a los riesgos del trasporte aéreo puesto que se encuentra en las dependencias del aeropuerto, mientras que tales peligros comienzan para él al ingresar en el área de partida, porque en ella evolucionan las aeronaves, tanto la que él debe tomar cuanto las demás que estén por partir o lleguen al termino de sus respectivos vuelos. Se encuentran comprendidos en este lapso los daños sufridos por el pasajero en el trayecto cumplido en el aeródromo para embarcar, es decir, cuando después de haber salido de los edificios del aeropuerto circula por las pistas destinadas a la evolución de las aeronaves. No están, por el contrario los hechos ocurridos en la aeroestación, ni el transporte pre y post aéreo, por cuanto se trata de operaciones distintas del transporte aéreo y no sujetas a los riesgos propios de éste...”), (el destacado me pertenece). (Obra citada, Tercera Edición, Ed. Zavalía, pág. 572.- “

"Por todo lo expuesto, entiendo que corresponde hacer lugar al recurso de apelación interpuesto por la parte actora y en consecuencia revocar el proveído de fecha 7 de noviembre de 2017 dictado por el señor Juez Federal No 1 de Córdoba en cuanto declaró la incompetencia del fuero federal, debiendo continuar la causa en el Tribunal de primera instancia. Sin costas atento a la naturaleza de la cuestión debatida. “

\section{FUENTES BIBLIOGRAFICAS}

GOMEZ, CLAUDIO DANIEL.Competencia Federal. Buenos Aires : Mediterranea.

HART, H. L.A.El Concepto de Derecho. Buenos Aires : Aberedo Perrot , 1961.

MARTINEZ MARTINEZ, ESUTAQUIO. Jurisprudencia Internacional sobre la Convencion de Valsovia de 1929, referente a la responsabilidad civil del porteador aéreo. [aut. libro] Goedhurs. Santiago de Chile : Editorial Juridica de Chile, 1962.

PALACIO, LINO ENRIQUE. Manual de Derecho Procesal Civil. Buenos Aires : LexisNexis - Abeledo - Perrot.

VIDELA ESCALADA, FEDERICO.Manual de Derecho Aeronautico. Buenos Aires: Zavalia, 2007. 


\section{JURISPRUDENCIA:}

Buronocore v. Trans World Airlines, Inc. 900 F.2d 8, 10, 2d Cir.. Estados Unidos : United States Court of Appeals, Second Circuit, 1990.

\section{PAGINAS WEBS:}

CAPALDO, GRISELDA. cedaeonline.com. [En línea] Powered by WordPress.com, 11 de 07 de 2013. [Citado el: 05 de 03 de 2019.] https://cedaeonline.com.ar/2013/07/11/nueve-anos-devigencia-del-convenio-de-montreal-de-1999-su-interpretacion-jurisprudencial-respecto. 